\title{
LA POÉSIE AU CYCLE 3. COMMENT ENSEIGNER UN ART EN MILIEU SCOLAIRE ?
}

\author{
Virginie Tellier
}

Armand Colin | « Le français aujourd'hui »

2018/3 N²02 | pages 81 à 91

ISSN 0184-7732

ISBN 9782200931858

DOI $10.3917 / \mathrm{lfa} .202 .0081$

\section{Article disponible en ligne à l'adresse :}

https://www.cairn.info/revue-le-francais-aujourd-hui-2018-3-page-81.htm

Distribution électronique Cairn.info pour Armand Colin.

(C) Armand Colin. Tous droits réservés pour tous pays.

La reproduction ou représentation de cet article, notamment par photocopie, n'est autorisée que dans les limites des conditions générales d'utilisation du site ou, le cas échéant, des conditions générales de la licence souscrite par votre établissement. Toute autre reproduction ou représentation, en tout ou partie, sous quelque forme et de quelque manière que ce soit, est interdite sauf accord préalable et écrit de l'éditeur, en dehors des cas prévus par la législation en vigueur en France. Il est précisé que son stockage dans une base de données est également interdit. 


\title{
LA POÉSIE AU CYCLE 3. COMMENT ENSEIGNER UN ART EN MILIEU SCOLAIRE?
}

\author{
Virginie TELLIER \\ Université de Cergy-Pontoise \\ Laboratoire «École, mutations, apprentissages » (ÉA 4507)
}

«L'enseignement de la poésie relève-t-il de la littérature?» Cette question, naïve en apparence, permet de mesurer l'écart entre les représentations dans l'enseignement primaire et dans l'enseignement secondaire. Un professeur de lettres répondrait sans ambigüité par l'affirmative : non seulement la poésie fait partie de la littérature, mais elle est même bien souvent considérée, à l'université, comme l'essence même de celle-ci. Un professeur des écoles hésiterait sans doute davantage. Car on "fait poésie», à l'école, depuis fort longtemps, bien avant que les programmes de 2002 n'aient formalisé ${ }^{1}$ l'enseignement de la littérature à l'école. L'examen des programmes de 2015 permet de mesurer l'enjeu que représente la création d'un "cycle 3 ", englobant les deux dernières années de l'école primaire et la première année d'école secondaire, si on s'efforce de mettre au jour les théories de référence qui sous-tendent ces programmes. Ce double éclairage, institutionnel et universitaire, confronté aux pratiques de classes et aux réticences des enseignants, permet de formuler quelques propositions didactiques pour créer les conditions de possibilité d'un véritable parcours poétique au sein du cycle qui permette, au travers de la pratique d'activités de lecture et d'écriture, de construire, chez les élèves, un rapport esthétique au monde.

\section{Poème, poésie, poétique}

La poésie a longtemps été, et reste encore dans beaucoup de classes, une "matière ", avec son corpus, son approche disciplinaire, ses exercices et son créneau dans l'emploi du temps hebdomadaire. Dans les programmes de 1995 pour l'enseignement primaire, l'usage poétique de la langue constituait un bloc spécifique, nettement détaché au sein de la discipline "français", au côté de l'oral, de la lecture, de l'écriture et de l'étude de la langue. Les programmes de 2002 et 2008 pour l'école primaire faisaient de la poésie une section du langage oral. Au collège, les programmes de 2008 réservaient une place particulière à la poésie, envisagée comme objet d'étude spécifique

1. On emploie ici le terme formaliser et non introduire, car la lecture de textes littéraires, à l'école, est une pratique continue depuis l'instauration de celle-ci. 
de la Sixième à la Troisième, selon des entrées que $S$. Larraburu-Dedouret (2010 : 31) pouvait juger «réductrices et partielles». Les programmes de 2015 pour le cycle 3 articulent les cultures didactiques de l'école et du collège, et ouvrent de nouvelles perspectives pour l'enseignement de la poésie à l'école.

\section{Copier, apprendre, réciter}

Ces programmes appréhendent encore la poésie comme une discipline scolaire. La «copie» de poèmes est ainsi envisagée à deux reprises (p. 104, p. 111), d'une part, comme activité destinée à accompagner la lecture, d'autre part, comme activité d'écriture. La poésie occupe également une place centrale dans l'enseignement du langage oral, tant en réception qu'en production. Il s'agit alors de maitriser les «techniques de mise en voix des textes littéraires» (p. 102). La dimension orale de la poésie est ainsi d'abord perçue comme l'occasion d'un travail technique : si la memoria et l'actio de la rhétorique classique sont bien présentes, on note l'absence de l'inventio, de la dispositio, et de l'elocutio, privilégiées d'ordinaire dans l'étude de la littérature, du moins au collège.

\section{Lire, écrire}

La seconde approche fait de la poésie un genre littéraire, dont l'étude relève essentiellement des compétences de lecture. Le programme des Cours moyens ( $4^{\text {ème }}$ et $5^{\text {ème }}$ primaire) propose une définition de la poésie : " comprendre que la poésie est une autre façon de dire le monde; dégager quelques-uns des traits récurrents et fondamentaux du langage poétique (exploration des ressources du langage, libertés envers la logique ordinaire, rôle des images, référent incertain, expression d'une sensibilité particulière et d'émotions)» (p. 109). Le programme de Sixième ( $1^{\text {ère }}$ du secondaire) invite à développer en outre des compétences d'analyse. Les traits définitoires de la poésie proposés pour le cycle 3 reprennent globalement les éléments essentiels du programme 2008 pour le collège. Les programmes de 2015 prolongent ainsi ceux de 2002, en continuant à déplacer vers l'école une dimension conceptuelle présente auparavant au collège : le développement des compétences d'interprétation des textes littéraires est désormais une dimension essentielle de l'enseignement de la littérature, dès l'école primaire. L'approche des textes poétiques doit néanmoins rester essentiellement intuitive, basée sur la réception du texte par l'élève, afin d'enrichir sa posture de lecteur, sans entrer trop avant dans une analyse technique des procédés d'écriture convoqués, démarche réservée pour les plus grandes classes. Parallèlement à la lecture de textes poétiques, les programmes recommandent également des activités d'écriture. 


\section{Créer : les "grandes entrées "}

Dans les grandes entrées pour la culture littéraire et artistique, l'étude de poèmes est envisagée en Cours moyen dans le cadre de "la morale en question" et "se découvrir, s'affirmer dans le rapport aux autres». On retrouve ici, en 2015, une continuité forte avec les programmes précédents, et notamment les programmes du collège de 2008, dans lesquels les Fables de La Fontaine faisaient l'objet d'une étude spécifique en Sixième, tandis que la poésie lyrique prenait place en Quatrième ( $3^{\text {ème }} \mathrm{du}$ secondaire) et la poésie engagée en Troisième ( $4^{\mathrm{ème}} \mathrm{du}$ secondaire). Ce qui parait plus novateur dans le cadre de ces deux entrées, c'est que les textes poétiques sont confrontés systématiquement à des textes appartenant à d'autres genres, et que leur lecture est facultative. On sent ainsi que la nature poétique du corpus est placée au second plan : c'est la dimension pragmatique et communicative qui doit guider le choix des textes et leur présentation aux élèves.

Deux entrées rendent obligatoire le recours à des corpus de textes poétiques, « imaginer, dire et célébrer le monde » en Cours moyen et " récits de création, création poétique» en Sixième. En Cours moyen, à partir de l'étude de poèmes et de contes étiologiques, on s'interroge sur le rapport entre l'homme et la nature. En Sixième, à partir de l'étude de poèmes et de récits de création, on s'interroge sur la conception du monde qui sous-tend les œuvres lues. La notion de genre est rendue secondaire par la mention «sans acception stricte de genre" (p. 122), au profit d'une étude qui insiste sur l'acte créateur, sur l'origine du monde, de l'homme et des sociétés.

\section{Fabriquer, transformer}

Les "grandes entrées » mettent ainsi l'accent sur l'étymon grec poïesis, à l'origine du latin poesis : la poésie est avant tout processus de fabrication, de création. Elle relève ainsi tout autant des arts plastiques que de la littérature. Les programmes de 2015 soulignent l'existence d'une visée "poétique » dans les démarches de création artistique. Ils portent ainsi un regard élargi sur la poésie, invitant enseignants et élèves à l'observer alors même que toute référence au langage articulé est absente. La poésie est alors, bien plus qu'un genre littéraire, un certain rapport au monde, qui ferait de "poétique " un quasi-synonyme d'" artistique».

\section{Théories de référence}

La place réservée à la poésie dans les programmes de 2015 du cycle 3 est complexe. Elle constitue une synthèse des approches plus anciennes qui avaient cours à l'école et au collège. Elle ajoute également une dimension nouvelle, qui déborde du cadre générique traditionnel. La poésie y est à la fois un genre littéraire spécifique, doté de ses caractéristiques propres, 
et littérarité vers laquelle tendent tous les textes littéraires, voire toutes les œuvres d'art.

\section{Le texte poétique}

L'approche qui domine dans les programmes scolaires depuis les années 1970 est centrée sur le texte, dans la lignée des travaux proposés par le formalisme russe. J.-F. Halté (1992 : 52) ironisait, dans l'ouvrage qu'il a consacré à la didactique du français, sur les transpositions du célèbre schéma de R. Jakobson dans les manuels scolaires : la référence y est souvent coupée du raisonnement dans lequel elle prend place. L'objectif est alors le développement chez les élèves de compétences de communication, comme le rappelle F. Sublet (1996). Vingt-cinq ans plus tard, la «fonction poétique "du langage reste une référence dans la didactique de la poésie à l'école, et dans la formation des enseignants du second degré. Cette conception explique également l'extension du corpus poétique à d'autres formes et d'autres genres, comme les slogans publicitaires ou les sketchs humoristiques : Raymond Devos est en passe de devenir un classique de l'étude du "langage poétique " à l'école. Tout jeu de mots est alors considéré comme poétique, au sens où il met l'accent sur la forme des mots.

On constate néanmoins aujourd'hui un infléchissement : la poésie n'est plus seulement mise au service du développement de compétences de communication, et commence à être davantage envisagée pour elle-même, en tant qu'art du langage. Le passage obligé par le «schéma » de R. Jakobson s'estompe, et on assiste à des relectures plus fidèles de son analyse de la "poéticité».

\section{Le sujet poétique}

D'autres théories, occultées par le structuralisme, trouvent aujourd'hui place dans les références de la didactique de la poésie. M. Bakhtine s'écarte du modèle formaliste par deux aspects au moins. D'une part, il souligne l'importance de l'intention créatrice, et partant l'historicité de tout poème ; d'autre part, il affirme l'interdépendance de la forme et du contenu, et déplace la question poétique de la linguistique vers l'esthétique. Pour autant, il maintient l'étroite relation entre poésie et langage : le langage est à la poésie ce que la couleur est à la peinture, son matériau.

Ce processus d'essentialisation est également très présent chez H. Meschonnic, dont la poétique du rythme est solidaire de la promotion de la notion de sujet: l'universalité de la parole poétique nait dans la création d'une relation intersubjective entre auteur et lecteur, qui transcende les individualités. Elle dépasse alors la notion d'intention d'auteur : le poème déborde toujours la volonté initiale du créateur. Sur le plan didactique, elle entre en résonnance avec les théories de la lecture et de la lecture littéraire. Les transpositions didactiques de ces théories sont davantage axées sur la réception des œuvres narratives, et ont donné lieu à 
de nombreux ouvrages s'intéressant à la compréhension et à l'interprétation des ouvres. C'est bien la perspective qui semble dominante dans les programmes de 2015, puisqu'il s'agit de "donner un sens à la lecture", comme le suggère le document d'accompagnement publié sur le site ÉDUSCOL du ministère de l'Éducation nationale. Celui-ci, après avoir montré les limites des approches historique et générique de la littérature, insiste sur les "fonctions de la lecture" et rattache l'enseignement de la poésie à la fonction «se servir du langage pour imaginer».

Qu'est-ce alors qu'être lecteur de poésie? Qu'est-ce que comprendre et interpréter un poème? Certaines des réponses qu'on peut apporter à ces questions sont vraisemblablement communes à tous les textes littéraires. Mais les analyses textuelles, en matière de poésie, nécessitent des connaissances linguistiques et culturelles, une maturité que les élèves de cycle 3 ne possèdent pas. Il apparait, à la lecture des textes d'accompagnement des programmes, que la spécificité de l'interprétation d'un texte poétique en cycle 3 réside essentiellement dans la capacité de l'élève à incarner le texte, à lui prêter son corps et sa voix. Cette dimension dépasse très largement le cadre assigné traditionnellement à la récitation de poèmes mémorisés : dans la lignée des programmes de 2002 et 2008, elle invite à faire de la diction poétique une authentique interprétation du texte, capable de donner corps au rythme du poème et de rendre manifeste l'intersubjectivité à l'œuvre.

\section{Le monde poétique}

Reste à envisager la question de la référence, et le rapport particulier que la parole poétique construit avec le monde. Cette dimension apparait comme essentielle dans les programmes de 2015, qui invitent à considérer que la poésie, comme les autres arts, est « une autre façon de dire le monde » (p. 109). Ceux-ci ne postulent jamais la gratuité des jeux langagiers à l'œuvre dans le langage poétique. Ils n'insistent pas non plus, comme c'était le cas dans les anciens programmes du collège, sur le lyrisme ou l'engagement de l'artiste : ces dimensions sont concédées à des entrées dans lesquelles le corpus poétique est secondaire par rapport au roman. Le verbe poétique, dans les entrées qui lui sont consacrées, est considéré dans un rapport de création avec le monde qu'il évoque ou convoque, qu'il soit imaginaire ou réel. Sans doute faut-il voir dans ce recentrement sur le monde l'une des manifestations de la préoccupation éthique - et secondairement écologique, fondamentale dans les programmes de 2015. Le programme de littérature est traversé par la question des valeurs, et l'étude de la poésie qu'il propose relève de ce même souci. On peut voir, ici encore, dans les travaux d'H. Meschonnic le fondement de cette conception de la poésie : son œuvre tend à étudier dans un même mouvement « la Rime et la vie ", affirmant que le structuralisme est responsable d'un triple " fiasco ", " celui de l'historicité, de la valeur, du sujet " (1989 : 110). Cet héritage permet d'expliquer l'importance grandissante, dans la didactique de la poésie, de la notion de "poème ", telle que la 
présente par exemple le $\mathrm{n}^{\circ} 169$ de la revue Le français aujourd'hui (2010) intitulé «Enseigner la poésie avec les poèmes ».

\section{Pratiques de classe}

Les programmes de 2015 conduisent à un déplacement des théories de référence pour l'enseignement de la littérature à l'école, marquant le déclin du structuralisme et la revendication du primat éthique, solidaire d'une centration sur le sujet lecteur. Se pose alors la question de savoir comment cette conception exigeante de la nature de la parole poétique peut être mise en œuvre dans les classes.

\section{Pratiques effectives}

À l'école, la poésie continue de constituer bien souvent une discipline à part entière, avec ses activités et ses codes. En témoigne la permanence des pratiques hebdomadaires - copie, mémorisation, illustration, récitation. L'analyse proposée par J.-L. Gaudet en 1995 du corpus poétique scolaire reste en grande partie d'actualité ${ }^{2}$. Parmi les auteurs classiques, La Fontaine et Hugo sont encore très largement étudiés dans les classes. Les auteurs du $\mathrm{XX}^{\mathrm{e}}$ siècle constituent un panthéon très peu soumis à variation : Prévert, Carême, Desnos sont lus et appris comme par le passé, et ce malgré le renouvèlement du corpus proposé dans les listes de référence. La succession des saisons et des fêtes continue de rythmer la progression des lectures dans la plupart des écoles.

La poésie, à l'école, participe aussi d'une poétisation de l'école. Ainsi en est-il des "Hiboux» de R. Desnos, qui visent à faire acquérir aux élèves une liste d'exceptions grammaticales, ou encore d' «En sortant de l'école » de J. Prévert, qui participe à la transfiguration poétique de l'expérience scolaire. La poésie manifeste ainsi l'un des pouvoirs de transmission et de permanence de l'institution. Si les enfants ne posent plus les multiplications comme leurs parents, s'ils n'apprennent plus la même grammaire que ces derniers, ils continuent d'interroger l'existence d'une « fourmi parlant français, parlant latin et javanais». Ainsi, si les enseignants expriment parfois leur lassitude face à des pratiques constamment répétées, d'autres soulignent également la puissance culturelle de l'exercice, et l'attachement au corpus de plusieurs générations de Français, dont l'expérience de la poésie se limite, pour beaucoup, à celle de l'enseignement reçu de trois à douze ans : on ne fait jamais autant poésie qu'à l'école. Les enseignants soulignent également le caractère sécurisant d'une pratique désormais ritualisée.

2. Notre propre analyse se fonde sur des discussions informelles avec des enseignants de l'Ain lors d'animations pédagogiques conduites en 2017-2018 et une enquête mise en œuvre par Nadège Besset et Catherine Cadoux dans le cadre de leur mémoire de Master 2, soutenu à l'ÉSPÉ de l'académie de Lyon (université Lyon 1 - Claude Bernard) le 8 juin 2018. Qu'elles en soient ici remerciées. 
Comme le montrait déjà M.-F. Berrendonner, en 1995, la pratique de la récitation, loin de disparaitre, s'est étendue au collège, où on continue d'apprendre par cœur des poèmes, tout au long de l'année. L'enseignement de la poésie y reste lui aussi traditionnel, fondé sur la lecture analytique, destinée à mettre au jour les caractéristiques - principalement formelles du genre. L'œuvre poétique reste principalement abordée dans le cadre de groupements de textes thématiques, choisis pour leur accessibilité et leur capacité à refléter l'entrée proposée : il s'agit ainsi d'enchanter le monde ou de créer des mondes. Dupés par les anciens intitulés des programmes de 2008, qui voyaient dans l'année de Sixième une année d' 'initiation à la poésie ", peu d'enseignants de collège ont une conscience véritable du travail effectué à l'école pour mettre en œuvre cette initiation.

Parmi les pratiques nouvelles, on notera l'importance accordée à la diction, en lieu et place de la récitation. Beaucoup d'enseignants invitent désormais les élèves à travailler leur mise en corps et en voix, à proposer plusieurs interprétations du texte étudié (chuchoter, crier, varier le rythme, slamer...). L'importance de former progressivement le gout et la posture des élèves lecteurs est également très largement prise en compte dans les classes, où les élèves sont de plus en plus souvent invités à effectuer leurs propres choix parmi des corpus plus larges, à proposer des textes découverts personnellement dans des anthologies ou à la maison.

L'écriture de textes poétiques tient également une place croissante dans les pratiques de classe. Dominent, certes, des activités très ritualisées et, pour certaines, peu créatives (jeux de langage soumis à des consignes très précises, écriture "à la manière de", reposant sur la reprise de patrons syntaxiques contraignants, comme les anaphores...). Pourtant, plus nombreux sont les enseignants à tenter des expériences artistiques d'ateliers d'écriture, laissant place à la libre création des élèves. En effet, l'expérience montre que les enfants, peu susceptibles, en cycle 3, de proposer ou même de comprendre des analyses stylistiques précises, manifestent une compréhension intuitive fine de ce qu'est la poésie, dès lors qu'on leur propose d'en écrire.

\section{Réticences}

La première réticence des enseignants du premier degré à l'égard de l'évolution des programmes tient au fait que, pour la plupart d'entre eux, la poésie ne s'enseigne pas : on peut initier les élèves, les sensibiliser, mais non leur transmettre des connaissances ${ }^{3}$. Le seul enseignement qu' il serait possible de délivrer est précisément celui avec lequel les programmes invitent à prendre de la distance : le travail répétitif sur les vers, les strophes, les rimes, la description de l'organisation matérielle du poème sur la page. On voit alors que l'idée d'un langage poétique sans poème est trop abstraite

3. Voir J.-L. Gaudet (op. cit. : 39-40). Le même constat a été opéré par Nadège Besset et Catherine Cadoux dans le cadre de leur enquête (cf. note 2). 
pour la plupart des enseignants, et excède leur capacité à faire évoluer leurs pratiques sans formation spécifique.

Beaucoup d'enseignants du premier degré peinent également à envisager que les modes de compréhension et d'interprétation des poèmes soient différents de ceux pratiqués pour les textes narratifs. Si beaucoup ont considérablement fait évoluer leurs pratiques pédagogiques relativement à la lecture de récits, peu sont ceux qui sont prêts à affronter les particularités des textes poétiques. Certains avouent trouver la poésie "trop difficile». D'autres manifestent leur réticence à proposer aux élèves des textes qu'euxmêmes "ne comprennent pas». Accepter la déroute du sens, perdre la maitrise du processus interprétatif à l'œuvre, voilà des difficultés auxquelles les enseignants se sentent confrontés, et qui expliquent le choix de corpus de poésie pour enfants, essentiellement ludiques, sans enjeu existentiel, incapables d'engager le lecteur dans un véritable processus interprétatif. Confrontés à la double impasse de considérer le poème comme un espace sacré indéchiffrable, qui souffrirait de toute analyse, ou comme un espace ludique, dont il serait possible de mettre les rouages et ressorts au jour, les enseignants peinent à trouver une autre voie. À cet égard, la proposition des programmes de 2015 de faire lire aux élèves des recueils entiers, conformément aux préconisations que formulait J.-P. Siméon en 1996, et que reprend le document "La poésie à l'école » publié sur ÉDUSCOL, n’a pas encore fait évoluer massivement les pratiques, faute d'une appropriation réelle de la démarche.

Les enseignants du second degré sont, pour la plupart, déroutés par le rapprochement opéré dans les programmes entre création poétique et création divine. Le choix opéré consiste généralement dans la constitution de deux séquences spécifiques, l'une portant sur les récits des origines, l'autre sur un corpus poétique mettant en valeur la création à l'œuvre dans le langage. Ce choix, conforté par les manuels, maintient donc la dominante générique dans l'enseignement secondaire : la poésie est un genre, défini essentiellement par des critères formels. Si les enseignants ont développé, depuis 2008, des compétences pour mettre en œuvre la comparaison entre les récits bibliques et les récits mythiques, le rapprochement avec le corpus poétique pose des problèmes : l'intention du texte poétique, le statut de son auteur, le schéma communicationnel qu'il met en place avec son lecteur constituent des différences qu'il est difficile de surmonter, pour des enseignants formés à l'analyse structurale du poème, plus que métaphysique.

Quant aux enseignants du premier degré, peu nombreux sont ceux qui ont envisagé frontalement cette grande entrée, parmi les six proposées. Le « conte étiologique " ne fait pas partie du corpus classique à l'école, et l'élaboration de séquences de poésie, dans le cadre de l'enseignement de la littérature, n'est pas encore, lui non plus, entré dans les pratiques courantes : enseignée sous forme de rituels toute l'année, la poésie est parfois mise à l'honneur lors du printemps des poètes, sans que des séquences pédagogiques soient 
encore clairement élaborées, avec des objectifs de compétences de lecture spécifiques.

On voit ici que le développement d'un enseignement de la poésie en cycle 3 nécessite une prise en compte des spécificités de celle-ci, dès la formation initiale des enseignants. Trois questions reviennent sans cesse : qu'est-ce que la poésie? que doit-on enseigner? que faire en classe? Seule la création de modules de formation spécifiques, conjoints aux futurs enseignants des premier et second degrés, pourrait sans doute permettre la mise en œuvre d'un parcours poétique cohérent en cycle 3. La mise en place, au sein des ÉSPÉ, d'ateliers de lecture orale, d'écriture et de diction des textes pourrait conduire à une modification des pratiques en vigueur. Comment demander à des enseignants qui ne lisent pas de poésie de faire lire des recueils à des élèves? Comment leur conseiller de mettre en place des ateliers d'écriture sans leur permettre de vivre cette expérience? Dans le premier degré, la formation des enseignants en matière de pratiques culturelles et artistique est encore trop insuffisante, faute de véritables projets culturels au sein des ÉSPÉ. Dans le second degré, les enseignants de lettres peinent à envisager la littérature comme un enseignement artistique, alors même qu'ils sont de plus en plus nombreux à conduire des projets culturels, notamment autour du cinéma. La poésie pose, plus encore que les autres genres littéraires, la question de la place dévolue aux arts à l'école. Le malaise enseignant est réel : si les enseignants se sentent aujourd'hui capables de transmettre des connaissances sur l'art, ils sont démunis lorsqu'il s'agit de faire vivre aux élèves une expérience artistique, qu'elle soit en réception ou en production.

\section{Propositions didactiques}

\section{Privilégier une approche sensible}

Le dossier "La poésie à l'école» soulevait avec pertinence la question épineuse de la compréhension en matière de poésie. S'il est admis aujourd'hui qu'on ne comprend pas un texte documentaire comme un récit de fiction, il importe également de préparer les enseignants à développer chez les élèves des compétences spécifiques en matière de lecture poétique. L'approche, en cycle 3, ne peut encore être analytique. Elle doit privilégier l'exposition aux textes, sans nécessairement envisager leur explication. Il s'agit bien de faire réagir les élèves, afin qu'ils s'approprient les textes, selon la pratique du " nourrissage » développée par le groupe ALEP (Favriaud et al. 2009) : lire et faire lire restent les deux impératifs de l'enseignement de la poésie en cycle 3. La phase de nourrissage précède une phase d'écriture créative, destinée à permettre à l'enfant de faire émerger progressivement une posture de sujet, qui commence par la construction d'un sujet narcissique, susceptible d'évoluer progressivement vers la constitution d'un sujet lyrique.

Cette approche sensible invite à reconsidérer le choix des textes à faire lire aux élèves. Les enseignants choisissent en effet souvent d'aborder la poésie à 
travers le plus grand nombre possibles de formes : on lit ainsi successivement des poèmes anciens et contemporains, en vers et en prose, courts et longs, narratifs et lyriques, afin de faire sentir aux élèves la diversité formelle du genre. L'intention - louable - est de faire émerger une représentation du poétique sans figer celle-ci. Il apparait pourtant à l'usage qu'un travail en intention est parfois plus efficace qu'un travail en extension. Autrement dit, la lecture d'un recueil de poèmes ou l'exploration d'un univers circonscrit permet de faire émerger une représentation de la parole poétique plus surement que la confrontation à une pluralité de textes divers. L'accent mis sur les activités de lecture et d'écriture repose sur l'acceptation, de la part de l'enseignant, de suspendre toute définition définitive, afin de permettre à chaque élève de construire son interrogation face aux textes et de former progressivement sa posture de lecteur.

\section{Articuler la poésie et les arts}

L'une des voies les plus fécondes ouverte par les programmes de 2015, et encore trop peu exploitée dans les classes, tant à l'école qu'au collège, semble être le rapprochement entre la poésie et les arts. L'usage de la chanson et des mises en musique de poèmes est une pratique déjà affirmée dans les classes, mais la musique y est bien souvent considérée comme aide à la mémoire, et non comme forme artistique spécifique. Parallèlement, si l'illustration des poèmes par le dessin est une pratique courante, pour ne pas dire constante dans les classes, si on invite sans cesse les élèves à repérer les « images» dans les poèmes, peu nombreux sont les enseignants qui croisent l'enseignement des arts plastiques avec celui de la poésie. En dépit des apparences, le cloisonnement disciplinaire est aussi fort dans l'enseignement primaire que dans l'enseignement secondaire. Les perspectives ouvertes par l'histoire des arts doivent encore être prolongées en matière de pratique des arts, de même que l'histoire culturelle doit progressivement laisser place à une authentique pratique culturelle. Confronter aux textes, susciter le désir de lire et d'écrire, amener progressivement à des pratiques et des rencontres susceptibles de déborder le cadre de l'établissement scolaire sont des objectifs difficiles, mais nécessaires. À ce titre, la poésie devrait permettre de contribuer efficacement à la mise en ouvre d'une véritable «éducation artistique et culturelle» en milieu scolaire.

On voit ainsi que, si les programmes de 2015 tiennent compte des principales recherches en didactique de la poésie de ces dernières années, ils ne rompent pas avec les pratiques antérieures, tant à l'école (pratique de la récitation) qu'au collège (approche générique). Le renouvèlement des pratiques nécessite une réflexion approfondie sur le "sujet lyrique", qui prolongerait celle qui a été menée sur le "sujet lecteur" pour les genres narratifs, en définissant de manière plus précise ce que signifie " comprendre et interpréter " un texte poétique. Il nécessite également que soient revalorisées les pratiques artistiques à l'école, afin de dégager, ne 
serait-ce que ponctuellement, les pratiques de lecture et d'écriture de leur finalité communicative pour en faire de véritables activités de création.

\section{Virginie TELLIER}

\section{Références bibliographiques}

- MINISTÈRE DE L'ÉDUCATION NATIONALE (2004). La Poésie à l'école. ÉDUSCOL, <http://cache.media.eduscol.education.fr/file/ecole/86/1/dossierpoesie_113861.pdf_.

- MINISTERE DE L'EDUCATION NATIONALE (2017). Imaginer, dire et célébrer le monde. Récits de création; création poétique. ÉDUSCOL, $<$ http://cache.media.eduscol.education.fr/file/Culture_litteraire_/52/7/RA16_

C3_FRA_Imaginer-dire-celebrer_Introduction_810527.pdf $>$.

- BERRENDONNER, M.-F. (1995). La poésie au collège. In J.-Y. Debreuille (dir.), Enseigner la poésie? (pp. 67-76). Lyon : Presses universitaires de Lyon.

- DUFAYS, J.-L., GEMENNE, L. \& LEDUR, D. (2015). Pour une lecture littéraire.

Histoire, théories, pistes pour la classe. Louvain-la-Neuve : De Boeck.

- FAVRIAUD, M., PLÉGAT-SOUTJIS, F., VINSONNEAU, M., ESCUILLIÉ, C., MIOSSEC, A., POLETTO, M., KAYSER, E., PANISSAL, N. \& DUTRAIT, C. (2009). Nourrissage, amorçage et gestes professionnels dans la production poétique au cycle 2 de l'école primaire. Repères, 40, 175-199.

- GAUDET, J.-L. (1995). La poésie à l'école, à quoi ça rime? In J.-Y. Debreuille (dir.), Enseigner la poésie? (pp. 37-66). Lyon : Presses universitaires de Lyon.

- HALTÉ, J.-F. (1992). La Didactique du français. Paris: Presses universitaires de France, coll. "Que sais-je?».

- LARRABURU-DEDOURET, S. (2010). De la poésie aux poèmes : quel questionnement du poétique dans le secondaire? Le français aujourd'hui, 169, 31-38.

- MESCHONNIC, H. (1989). La Rime et la vie. Paris : Verdier.

- SIMÉON, J.-P. (1996). Lecture de la poésie à l'école primaire. Une démarche possible : la lecture d'une œuvre poétique complète. Repères, 13, 131-146.

- SUBLET, F. (1996). Au vif des mots, au cœur des choses. Recherches INRP en poésie dans les années 70. Repères, 13, 103-129. 